\title{
On the Equivalence of Different Order Parameters and Coexistence of Phases for Ising Ferromagnet. II
}

\author{
C. Gruber \\ Ecole Polytechniđúe
}

\section{J. L. Lebowitz}

Department of Mathematics, Rutgers University, New Brunswick, New Jersey 08903, USA

\begin{abstract}
We investigate' Ising spin systems with general ferromagnetic, translation invariant interactions, $H=-\sum J_{B} \sigma_{B}, J_{B} \geqq 0$. We show that the critical temperature $T_{i}$ for the order parameter $p_{i}$ defined as the temperature below which $p_{i}>0$, is independent of the way in which the symmetry breaking interactions approach zero from above. Furthermore, all the "equivalent" correlation functions have the same critical exponents as $T \rightarrow T_{i}$ from below, e.g. for pair interactions all the odd correlations have the same critical index as the spontaneous magnetization. The number of fluid and crystalline phases (periodic equilibrium states) coexisting at a temperature $T$ at which the energy is continuous is shown to be related to the number of symmetries of the interactions. This generalizes previous results for Ising spins with even (and non-vanishing nearest-neighbour) ferromagnetic interactions. We discuss some applications of these results to the triangular lattice with three body interactions and to the Ashkin-Teller model. Our results give the answer to the question raised by R.J. Baxter et al. concerning the equality of some critical exponents.
\end{abstract}

\section{Introduction}

In two recent articles [1,2], one of us obtained new results on the "equivalence of different order parameters" and "coexistence of phases" for Ising ferromagnets. These results were derived for systems with even interactions which "generate" all even subsets of the lattice, e.g. when the nearest neighbour interaction does not vanish for a simple Bravais lattice.

The purpose of this note is to draw attention to the fact that these results can be extended immediately to arbitrary interactions using the group structure associated with lattice systems [3]. The main new features which appear in the general case is the possibility of several critical temperatures $T_{i}$ each of which is associated with an order parameter $p_{i}$. We shall show that the different order parameters belonging to the same "equivalence class" as $p_{i}$ define the same critical

* Supported by NSF Grant PHY 77-22302 
temperature $T_{i}$ and have the same critical exponent as $p_{i}$; furthermore, the critical temperature $T_{i}$ which is defined as the temperature below which $p_{i}$ does not vanish is independent of the way the symmetry breaking interactions approaches zero from above.

It is further shown that at all temperatures where the energy is continuous (as a function of temperature), there exists a unique translation invariant equilibrium state which has the same internal symmetry group as the hamiltonian and is called the "Invariant Equilibrium State". From this last property follows that there coexist exactly $\left|\mathscr{S} / \mathscr{S}_{+}\right|$distinct periodic extremal equilibrium state, where $\mathscr{S}$ and $\mathscr{S}_{+}$are respectively the internal symmetry group of the hamiltonian and of the state $\omega_{+}$obtained by means of the $(+)$boundary conditions; $|\mathscr{S}| \mathscr{S}_{+} \mid$is the number of elements in the quotient group. While $\mathscr{S}$ depends on the hamiltonian $\mathscr{S}_{+}$will generally depend also on the temperature, e.g. for the Ising system on the square lattice with nearest neighbor pair interactions in zero magnetic field, $\mathscr{S}$ consists of the identity and spin reversal, $|\mathscr{S}|=2$, while $\mathscr{S}_{+}=\mathscr{S}$ for $T \geqq T_{c}$ the critical temperature but is reduced to the identity for $T<T_{c}$ and $|\mathscr{S}| \mathscr{S}_{+} \mid=2$. (We assume $|\mathscr{P}|$ finite.) It should be noted however that these distinct states are not necessarily distinct pure phase since some may not be invariant under the full translation group.

The interest of our results will be illustrated with some examples which are not covered by the conditions of [1,2]. An extension of the results of $[1,2]$ to continuous one component spin systems has been given in [4].

\section{Definitions and Properties of Lattice Systems}

We recall that a spin $\frac{1}{2}$ lattice system $\{\mathscr{L}, K, \mathscr{B}\}$ is defined by a "Lattice $\mathscr{L} "$ accountable set of points in $\mathbb{R}^{v}$ - a real function $K$ defined on $\mathscr{P}_{f}(\mathscr{L})$ - the finite subsets of $\mathscr{L}$-describing the "interactions", and $\mathscr{B}$ is the family of "bonds" defined as those subsets $B$ of $\mathscr{L}$ such that $K(B) \neq 0$. The configuration space of the system is taken to be the group $\mathscr{P}(\mathscr{L})$ of subsets of $\mathscr{L}$ with the product defined by the symmetric difference: $X \cdot Y=X \cup Y \backslash X \cap Y$ for $X, Y \subset \mathscr{L}$ i.e. $X, Y \in \mathscr{P}(\mathscr{L})$. We associate with any $X \in \mathscr{P}_{f}(\mathscr{L})$ the function $\sigma_{X}$ on $\mathscr{P}(\mathscr{L})$ defined by $\sigma_{X}(Y)=(-1)^{|X \cap Y|}$, with $|Z|=$ cardinality of the set $Z$. In the spin terminology a configuration $Y$ specifies the set of sites $y \in \mathscr{L}$ at which $\sigma_{y}=-1$ and $\sigma_{X}=\prod_{x \in X} \sigma_{x}$.

For any finite subset $\Lambda$ of $\mathscr{L}$, the Gibbs State $\omega_{\Lambda ; Y}$ of the finite system $\Lambda$ with boundary condition $Y \in \mathscr{P}(\mathscr{L})$ is defined by the probability measure on $\mathscr{P}(\Lambda)$ given by :

$$
\begin{aligned}
& \mu_{(\Lambda ; Y)}(X)=Z(\Lambda ; Y)^{-1} \exp \left[\sum_{B \in \mathscr{B}{ }_{\Lambda}} K(B) \sigma_{B}\left(Y^{c} X\right)\right] \\
& \mathscr{B}_{\Lambda}=\{B \in \mathscr{B} ; B \cap \Lambda \neq \phi\} \quad Y^{c}=Y \cap(\mathscr{L} \backslash \Lambda)
\end{aligned}
$$

i.e. $Y^{c}$ specifies the set of spins outside $\Lambda$ which are pointing down and $\mu_{(\Lambda ; Y)}(X)$ is the probability of a configuration in $\Lambda$ such that the spins in $X \subset A$ are pointing down.

Notice that the inverse temperature $\beta=\frac{1}{k T}$ has been introduced in the function $K$, i.e. $K=\beta J$, where $J$ is the effective interaction. 
The Gibbs State $\omega_{\Lambda ; 0}$ of the finite system $\Lambda$ with open (zero) boundary condition is defined by the probability measure on $\mathscr{P}(\Lambda)$ given by:

$$
\mu_{(\Lambda ; 0)}(X)=Z(\Lambda ; 0)^{-1} \exp \left[\sum_{\substack{B \in \mathscr{B} \\ B \subset A}} K(B) \sigma_{B}(X)\right] .
$$

Limits $\Lambda \rightarrow \mathscr{L}$ determine Gibbs (equilibrium) states $\omega$ on $P(\mathscr{L})$. In the following the states are defined by the correlation functions $\omega\left[\sigma_{X}\right], X \in \mathscr{P}_{f}(\mathscr{L})$; furthermore the group $\mathscr{P}(\mathscr{L})$ acts as a group of transformations on the states in the following manner:

$$
\forall Z \in \mathscr{P}(\mathscr{L}) \quad\left(\tau_{Z}^{\prime} \omega\right)\left[\sigma_{X}\right]=\sigma_{X}(Z) \quad \omega\left[\sigma_{X}\right] .
$$

The internal symmetry group $\mathscr{S}$ is the subgroup of $\mathscr{P}(\mathscr{L})$ defined by

$$
\mathscr{S}=\left\{S \subset \mathscr{L} ; \sigma_{B}(S)=+1 \forall B \in \mathscr{B}\right\}
$$

and has the following property [3]:

"For any [extremal] equilibrium state $\omega$ of $\{\mathscr{L}, K, \mathscr{B}\}$, and any $S$ in $\mathscr{S}$ the state $\tau_{S}^{\prime} \omega$ is also an [extremal] equilibrium state of $\{\mathscr{L}, K, \mathscr{B}\} . "$

The state is said to be "symmetric" if it is invariant under the internal symmetry group, i.e. $\tau_{S}^{\prime} \omega=\omega$ for all $S$ in $\mathscr{S}$. In fact, $\omega$ is symmetric if and only if $\omega\left[\sigma_{X}\right]=0$ for all $X \notin \overline{\mathscr{B}}$ where $\overline{\mathscr{B}}$ is the subgroup of $\mathscr{P}_{f}(\mathscr{L})$ generated by (finite products of bonds $B$ in) $\mathscr{B}$. It thus follows that if $\omega$ is not symmetric then there exists at least two distinct equilibrium states of the system namely $\omega$ and $\tau_{S}^{\prime} \omega$ for some $S$ in $\mathscr{S}$.

In the following we shall consider only ferromagnetic systems with $\mathbb{Z}^{\nu}$ invariant, finite range interactions, i.e.

$$
\begin{array}{lll}
x+a \in \mathscr{L} \text { for all } \quad x \in \mathscr{L} \text { and } & a \in \mathbb{Z}^{v} \\
K(B)=K(B+a) \geqq 0 & \\
K(B)=0 \quad \text { if Diameter } \quad B>R & (R<\infty)
\end{array}
$$

and for such systems we have the following result ([3, p. 65], [8]).

"With $\omega_{+}$the extremal, $\mathbb{Z}^{v}$-invariant equilibrium states obtained by means of the $(+)$ boundary conditions $(\mathrm{Y}=\phi)$ and $\mathscr{S}_{+}$the internal symmetry group of the state $\omega_{+}$defined by:

$$
\mathscr{S}_{+}=\left\{S \in \mathscr{S} ; \tau_{S}^{\prime} \omega_{+}=\omega_{+}\right\}
$$

then there exist at least $\left|\mathscr{S} / \mathscr{S}_{+}\right|$distinct extremal equilibrium states given by $\tau_{S}^{\prime} \omega_{+}$ with $S \in \mathscr{S} / \mathscr{S}_{+}$. Moreover if there exists a unique translation invariant, symmetric equilibrium state, then there exists exactly $\left|\mathscr{S} / \mathscr{S}_{+}\right|$distinct extremal equilibrium states which are invariant under some subgroup $\mathscr{T}$ of the translation group $\mathbb{Z}^{v}$ with $\mathbb{Z}^{v} / \mathscr{T}$ finite; furthermore

$$
\omega_{0}=\left.|\mathscr{S}| \mathscr{S}_{+}\right|^{-1} \sum_{S \in \mathscr{S} \mid \mathscr{S}_{+}} \tau_{S}^{\prime} \omega_{+} \cdot "
$$

We recall that the uniqueness of the invariant equilibrium states has been proved under very general conditions at high and low temperatures; it has also been shown [3] that $\left|\mathscr{S}_{+}\right|=|\mathscr{S}|$ and $\left|\mathscr{S}_{+}\right|=1$ respectively at high and low temperatures ${ }^{1}$.

$1 \quad\left|\mathscr{S}_{+}\right|=1$ at low temperature for $v \geqq 2$ and requires in general $|\mathscr{S}|$ finite 
The results which we shall obtain extend the results concerning the uniqueness of the invariant equilibrium state to all temperatures $T$ where the energy is a continuous function of $T$.

To conclude this summary, we remark that the group $\overline{\mathscr{B}}$ generalises to arbitrary interactions the even sets of $[1,2]$; the group of transformations $\tau_{S}^{\prime}, S \in \mathscr{S}$, generalise the spin reversal transformations; the family $\left\{\tau_{S}^{\prime} \omega_{+}\right\}_{S \in \mathscr{S} \mid \mathscr{S}_{+}}$of equilibrium states generalises $\left\{\omega_{+}, \omega_{-}=\tau_{\mathbb{Z}^{\nu}}^{\prime} \omega_{+}\right\}$and Equation (3) generalises the equation $\omega_{0}=\frac{1}{2}\left(\omega_{+}+\omega_{-}\right)$.

\section{On the Equivalence of Order Parameters for Ising Ferromagnets}

Let $\{\mathscr{L}, K=\beta J, \mathscr{B}\}$ be a $\mathbb{Z}^{v}$-invariant ferromagnetic lattice system as defined in Section 2 and let us introduce the decomposition of the group $\mathscr{P}_{f}(\mathscr{L})$ induced by the subgroup $\overline{\mathscr{B}}$, i.e.

$$
\mathscr{P}_{f}(\mathscr{L})=\bigcup_{i=1}^{\mid \mathscr{S}} X_{i} \overline{\mathscr{B}}, \quad X_{1}=\phi, \quad X_{i} \notin \overline{\mathscr{B}} \quad i>1
$$

(which generalises the decomposition of $\mathscr{P}_{f}(\mathscr{L})$ into even and odd subsets introduced in [1]).

The purpose of this section is to obtain informations about the critical exponents of $\left\langle\sigma_{X_{i} \bar{B}}\right\rangle, i>1, \bar{B} \in \overline{\mathscr{B}}$. To do so, we shall follow exactly [1] indicating the modifications necessary to extend those results to the general case.

The basic inequality which is the starting point of [1] and which follows from the Griffiths, Kelley, Sherman GKS-inequality [5] becomes in our case:

$$
k_{\bar{B}}\left\langle\sigma_{X}\right\rangle \leqq\left\langle\sigma_{X \bar{B}}\right\rangle \leqq k_{\bar{B}}^{-1}\left\langle\sigma_{X}\right\rangle
$$

where $k_{\bar{B}}$ can be taken independant of $T$ for all $T \leqq T_{0}$. In fact, with $\bar{B}=\prod_{i=1}^{n} B_{i}$ we can take

$$
k_{\bar{B}}=\prod_{i=1}^{n} \tanh \left(\beta_{0} J\left(B_{i}\right)\right) .
$$

For ferromagnetic systems the infinite volume limit of Gibbs states with open or positive boundary conditions exists and are $\mathbb{Z}^{v}$-invariant [6]; we denote these states by $\omega_{(\beta, J ; \alpha)}, \alpha=0,+$. Letting $\boldsymbol{J}^{\prime}=\left\{J_{i}^{\prime}\right\}_{i=1,2, \ldots|\mathcal{P}|}$ where $J_{1}^{\prime}=J$ and $J_{i}^{\prime} i \neq 1$ is a function on $X_{i} \mathscr{\mathscr { B }}$ [Eq. (4)], we have for all $S$ in $\mathscr{S}$ :

$$
\omega_{\left(\beta, \boldsymbol{J}^{\prime} ; \alpha\right)}\left[\sigma_{X}\right]=\sigma_{X}(S) \omega_{\left(\beta, \tau S^{\prime} ; \bar{\alpha}\right)}\left[\sigma_{X}\right]
$$

where

$$
\begin{aligned}
& \left(\tau_{S} J^{\prime}\right)_{1}=J \quad\left(\tau_{S} J^{\prime}\right)_{i}=\sigma_{X_{i}}(S) J_{i}^{\prime} \\
& \bar{\alpha}=0 \quad \text { if } \quad \alpha=0 \\
& \bar{\alpha}=S \text { if } \quad \alpha=+
\end{aligned}
$$


and $\omega_{\left(\beta, \tau_{S} J^{\prime} ; \bar{\alpha}\right)}$ is the Gibbs state with interactions $\tau_{S} J^{\prime}$ and boundary conditions $S$ or $(0)$.

We now consider the behaviour of the correlation functions $\omega_{\left(\beta, \mathbf{J}^{\prime} ; \alpha\right)}\left[\sigma_{X_{i} \bar{B}}\right]$ in the limit where the $J_{i}^{\prime}$ 's tend to zero for $i>1$ while $J_{1}^{\prime}=J$ remains fixed. To answer this question, we recall the following lemmas given in [1]:

Lemma 1. Let $\mathbf{J}^{\prime} \geqq 0$ then

(i) $\lim _{\boldsymbol{J}^{\prime} \rightarrow J} \omega_{\left(\beta, J^{\prime} ; 0\right)}\left[\sigma_{X}\right]=\omega_{(\beta, J ; 0)}\left[\sigma_{X}\right]$.

(ii) $\lim _{\mathbf{J}^{\prime}>J} \omega_{\left(\beta, \boldsymbol{J}^{\prime} ;+\right)}\left[\sigma_{X}\right]=\omega_{\left(\beta, J^{\prime+}\right)}\left[\sigma_{X}\right]$.

Lemma 2. Let $\mathbf{J}^{\prime}=\left(J_{X}^{\prime}, \hat{J}\right)$ where $J_{X}^{\prime}=J^{\prime}(X+a)>0, \hat{J} \geqq 0$, then

(i) $\omega_{\left(\beta, J_{X}, \hat{j} ; 0\right)}\left[\sigma_{X}\right]=\lim _{J_{X}>J_{X}} \frac{\partial \psi\left(J_{X}^{\prime}, \hat{J}\right)}{\partial J_{X}^{\prime}}$

$$
=\lim _{\left.J_{X}^{\prime}\right\rangle^{\prime} J_{X}} \omega_{\left(\beta, J_{X}^{\prime}, \hat{J}_{;}+\right)}\left[\sigma_{X}\right] \text { for all } X \in \mathscr{B} ;
$$

(ii) $\omega_{\left(\beta, J_{X}^{\prime}, \hat{J} ;+\right)}\left[\sigma_{X}\right]=\lim _{J_{X} \backslash J_{X}} \frac{\partial \psi\left(J_{X}^{\prime}, \hat{J}\right)}{\partial J_{X}^{\prime}}$

$$
=\lim _{J_{X}^{\prime}>J_{X}} \omega_{(\beta, J \dot{X}, \hat{j} ; 0)}\left[\sigma_{X}\right] \text { for all } X \in \mathscr{P}_{f}(\mathscr{L}),
$$

where $\psi=\beta^{-1} \lim _{\Lambda \rightarrow \mathscr{L}} \frac{\ln Z(\Lambda)}{|\Lambda|}$, the free energy per site, is independant of boundary condition.

Remark. We should stress that in Lemma 1 the limit $\boldsymbol{J}^{\prime} \rightarrow J$ can be taken along any "path"; on the other hand in Lemma 2, to study $\omega\left[\sigma_{X}\right]$ we must take $J_{X}^{\prime}>0$ and consider the limit $J_{X}^{\prime} \rightarrow J_{X}$. In particular this implies that if $\boldsymbol{J}^{\prime}=\left(J, J_{X_{i}}^{\prime}\right) i>1$ then

$$
\lim _{J_{\dot{X}_{i}}>0} \omega_{\left(\beta, J^{\prime} ;+\right)}\left[\sigma_{X_{i}}\right]=\lim _{J_{\dot{X}_{i}}>0} \omega_{\left(\beta, J^{\prime} ; 0\right)}\left[\sigma_{X_{i}}\right]=\omega_{(\beta, J ;+)}\left[\sigma_{X_{i}}\right]
$$

however we can not conclude for any $\bar{B}$ that the following order parameters are equal,

$$
\begin{aligned}
& \omega_{(\beta, J ;+)}^{*}\left[\sigma_{X_{i} \bar{B}}\right]=\lim _{J_{\dot{X}_{i}>0}} \omega_{\left(\beta, \boldsymbol{J}^{\prime} ;+\right)}\left[\sigma_{X_{i} \bar{B}}\right]=\omega_{(\beta, J ;+)}\left[\sigma_{X_{i}}\right] \\
& \omega_{(\beta, J ; 0)}^{*}\left[\sigma_{X_{i} \bar{B}}\right]=\lim _{\boldsymbol{X}_{\dot{X}_{i}>0}} \omega_{\left(\beta, \boldsymbol{J}^{\prime} ; 0\right)}\left[\sigma_{X_{i} \bar{B}}\right] .
\end{aligned}
$$

Let us now first consider the case where $J^{\prime}=\left(J, J_{i}^{\prime}\right)$ with $J_{i}^{\prime}=J^{\prime}\left(X_{i}+a\right)$; we can define for given fixed $J$ the "order parameter $p_{i}(\beta)$ " as

$$
p_{i}(\beta)=\lim _{J_{i}>0} \frac{\partial \psi\left(J, J_{i}^{\prime}\right)}{\partial J_{X}^{\prime}}=\lim _{J_{i}^{\prime}>0} \omega_{\left(\beta, J, J_{i}^{\prime} ; \alpha\right)}\left[\sigma_{X_{i}}\right]=\omega_{(\beta, J ;+)}\left[\sigma_{X_{i}}\right]
$$

and introduce a "critical temperature $\beta_{i}^{-1}$ for the order parameter $p_{i}$ " by

$$
p_{i}(\beta)=\left\{\begin{array}{rr}
0 & \beta<\beta_{i} \\
>0 & \beta>\beta_{i}
\end{array}\right.
$$


together with a "critical temperature $\beta_{c}$ " defined by

$$
\beta_{c}=\min \left\{\beta_{i}\right\} \text {. }
$$

The following lemma follows at once from the basic inequality Equation (5).

Lemma 3. Let $\boldsymbol{J}^{\prime}=\left(J, J_{i}^{\prime}\right)$ be the $\mathbb{Z}^{v}$-invariant ferromagnetic interaction introduced above and define

$$
\omega_{(\beta, J ; \alpha)}^{*}\left[\sigma_{X_{i} \bar{B}}\right]=\lim _{J_{i} \rtimes 0} \omega_{\left(\beta, J^{\prime} ; \alpha\right)}\left[\sigma_{X_{i} \bar{B}}\right]
$$

then for all $\bar{B} \in \overline{\mathscr{B}}$

$$
\begin{aligned}
& K_{i, 1} p_{i}(\beta) \leqq \omega_{(\beta, J ; \alpha)}^{*}\left[\sigma_{X_{i} \bar{B}}\right] \leqq K_{i, 2} p_{i}(\beta) \\
& 0<K_{i, 1}, K_{i, 2}<\infty
\end{aligned}
$$

In particular :

$$
\omega_{(\beta, J ; \alpha)}^{*}\left[\sigma_{X_{i} \bar{B}}\right]=0 \text { iff } p_{i}(\beta)=0
$$

and the behaviour

$$
p_{i}(\beta) \sim\left(\beta-\beta_{i}\right)^{b_{i}} \text { for } \quad \beta \geqq \beta_{i}
$$

implies

$$
\omega_{(\beta, J ; \alpha)}^{*}\left[\sigma_{X_{i} \bar{B}}\right] \sim\left(\beta-\beta_{i}\right)^{b_{i}} \text { for } \beta \geqq \beta_{i} .
$$

Lemma 4. For $\beta<\beta_{i}$ all equilibrium states have vanishing correlation functions on $X_{i} \overline{\mathscr{B}}$ if $J_{i}^{\prime}=0$.

Indeed, for any boundary condition $Y$, we have the inequality

$$
\omega_{\left(\beta, J, J_{i}^{\prime} ; Y\right)}\left[\sigma_{X_{i} \bar{B}}\right] \leqq \omega_{\left(\beta, J,\left|J_{i}^{\prime}\right| ;+\right)}\left[\sigma_{X_{i} \bar{B}}\right]
$$

then

$$
\lim _{J_{i}^{\prime} \rightarrow 0} \omega_{\left(\beta, J, J_{i}^{\prime} ; Y\right)}\left[\sigma_{X_{i} \bar{B}}\right] \leqq 0 \quad \text { if } \quad \beta<\beta_{i} \forall Y .
$$

Using then the symmetry relation Equation (6) implies that for some $S \in \mathscr{S}$

$$
-\omega_{\left(\beta, J, J_{i}^{i} ; Y\right)}\left[\sigma_{X_{i} \bar{B}}\right]=\omega_{\left(\beta, J,-J_{i} ; S Y\right)}\left[\sigma_{X_{i} \bar{B}}\right]
$$

and thus

$$
\lim _{J_{i}^{\prime} \rightarrow 0} \omega_{\left(\beta, J, J_{i}^{\prime} ; Y\right)}\left[\sigma_{X_{i} \bar{B}}\right]=0 \quad \text { if } \quad \beta<\beta_{i}
$$

for any boundary condition $Y$.

Finally let us also introduce the "zero- $J_{i}$ susceptibility"

$$
\chi_{(i, \alpha)}(\beta, A)=\lim _{J_{i}^{\prime} \rtimes 0} K_{i}^{\prime-1}\left(\omega_{\left(\beta, J, J_{i}^{\prime} ; \alpha\right)}\left[\sigma_{A}\right]-\omega_{(\beta, J ; \alpha)}^{*}\left[\sigma_{A}\right]\right)
$$


it then follows from the above lemmas and discussion that if

$$
\chi_{(i ; \alpha)}\left(\beta, X_{i}\right) \sim\left(\beta_{i}-\beta\right)^{-\gamma_{i}} \quad \beta \leqq \beta_{i}
$$

then

$$
\chi_{(i ; \alpha)}\left(\beta, X_{i} \bar{B}\right) \sim\left(\beta_{i}-\beta\right)^{-\gamma_{i}} \quad \beta \leqq \beta_{i}
$$

where $\gamma_{i}$ is independent of $\alpha=0,+$ and independant of $\bar{B} \in \overline{\mathscr{B}}$.

The choice of $J_{i}^{\prime}=J^{\prime}\left(X_{i}\right)$ as the sole symmetry breaking interaction is of course arbitrary; it follows however from the above lemmas and the remark following Lemma 3 that the critical temperature $\beta_{i}$ is unique independant of the way the symmetry breaking interaction defined on $X_{i} \overline{\mathscr{B}}$ tends to zero.

Theorem 5. Let $\{\mathscr{L}, K=\beta J, \mathscr{B}\}$ be a $\mathbb{Z}^{v}$-invariant, ferromagnetic system and $\mathscr{P}_{f}(\mathscr{L})=\bigcup_{i} X_{i} \overline{\mathscr{B}}, X_{1}=\phi, X_{i} \notin \overline{\mathscr{B}}$ for $i>1$, be the decomposition of $\mathscr{P}_{f}(\mathscr{L})$ induced by $\overline{\mathscr{B}}$ the subgroup of $\mathscr{P}_{f}(\mathscr{L})$ generated by finite products of bounds $B$ in $\mathscr{B}$. Then,

a) There exist $\beta_{i}, 1=2, \ldots|\mathscr{S}|$, not necessarily distinct, and positive constants $C_{i, 1}, C_{i, 2}$ such that for all $\bar{B} \in \overline{\mathscr{B}}$

$$
\omega_{(\beta, J ;+)}\left[\sigma_{X_{i} \bar{B}}\right]=\left\{\begin{array}{rr}
0 & \beta<\beta_{i} \\
>0 & \beta>\beta_{i}
\end{array}\right.
$$

and

$$
C_{i, 1} p_{i}(\beta) \leqq \omega_{(\beta, J ;+)}\left[\sigma_{X_{i} \bar{B}}\right] \leqq C_{i, 2} p_{i}(\beta)
$$

where

$$
p_{i}(\beta)=\omega_{\left(\beta, J^{++}\right)}\left[\sigma_{X_{i}}\right] .
$$

b) Let $J_{i}^{\prime} \geqq 0$ be $\mathbb{Z}^{v}$-invariant interactions defined on $X_{i} \overline{\mathscr{B}}, i>1$, then for $\boldsymbol{J}^{\prime}=\left\{J_{i}^{\prime}\right\}$ $J_{1}^{\prime}=J$ fixed and $\bar{B} \in \overline{\mathscr{B}}$

$$
\begin{aligned}
\omega_{(\beta, J ;+)}\left[\sigma_{X_{i} \bar{B}}\right] & =\lim _{\substack{J_{i}^{\prime}>0 \\
i \neq 1}} \omega_{\left(\beta, \boldsymbol{J}^{\prime} ;+\right)}\left[\sigma_{X_{i} \bar{B}}\right] \\
& =\lim _{\substack{J_{i}^{\prime}>0 \\
i \neq 1}} \frac{\partial \psi\left(\boldsymbol{J}^{\prime}\right)}{\partial J^{\prime}\left(X_{i} \bar{B}\right)}
\end{aligned}
$$

where the last equality holds when $J^{\prime}\left(X_{i} \bar{B}\right)>0$.

c) For $\beta<\beta_{i}$ all infinite volume equilibrium states of $\{\mathscr{L}, K, \mathscr{B}\}$ have correlations functions which vanish on $X_{i} \overline{\mathscr{B}}$.

For $\beta<\beta_{c}=\min \left\{\beta_{i}\right\}$ all infinite volume equilibrium state of $\{\mathscr{L}, K, \mathscr{B}\}$ are symmetric.

d) If $J_{i}^{\prime}=J^{\prime}\left(X_{i} \bar{B}\right)>0, i \neq 1 \quad \bar{B} \in \overline{\mathscr{B}}$ then for any $A \in X_{i} \overline{\mathscr{B}}$

$$
\chi_{X_{i} \bar{B}}(\beta, A ;+)=\limsup _{\boldsymbol{J}^{\prime} \rtimes J}{J_{i}^{\prime}}^{-1} \omega_{\left(\beta, \mathbf{J}^{\prime} ;+\right)}\left[\sigma_{A}\right]
$$

satisfies the inequalities

$$
C_{i, 1} \chi_{X_{i} \bar{B}}\left(\beta, X_{i} ;+\right) \leqq \chi_{X_{i} \bar{B}}(\beta, A ;+) \leqq C_{i, 2} \chi_{X_{i} \bar{B}}\left(\beta, X_{i} ;+\right)
$$


where however the values of $\chi_{X_{i} \bar{B}}$ may depends on the path $\mathbf{J}^{\prime}>J$.

Corollary 6. Let $\{\mathscr{L}, C, K, \mathscr{B}\}$ be a $\mathbb{Z}^{v}$-invariant ferromagnetic system with constraints satisfying the subgroup property, then for all $\bar{B} \in \mathscr{\mathscr { B }}$

$$
\omega_{(\beta ; 0)}\left[\sigma_{\bar{B}}\right]=\lim _{K_{B_{\infty}}^{\prime} \rightarrow+\infty} \omega_{\left(\beta, K^{\prime} ; 0\right)}\left[\sigma_{\bar{B}}\right]
$$

i.e. the symmetric correlation functions associated with open boundary condition are the limit of the correlations for a system without constraints with some interaction becoming infinite.

Indeed for systems such that the family of admissible configurations is a subgroup of $\mathscr{P}(\mathscr{L})$ the constraints are of the form [3]

$$
C_{B_{\infty}}(X)=\frac{1}{2}\left(1+\sigma_{B_{\infty}}(X)\right) \quad B_{\infty} \in \mathscr{B}_{\infty} \subset \mathscr{P}_{f}(\mathscr{L})
$$

i.e.

$$
C_{B_{\infty}}(X)=\lim _{K_{\mathbf{B}_{\infty} \rightarrow+\infty}} \exp \left[K_{B_{\infty}}^{\prime}\left(\sigma_{B_{\infty}}(X)-1\right)\right] .
$$

By duality argument [3] the state $\omega_{(\beta, K ; 0)}$ is related to the state $\omega_{\left(\beta, K^{*} ;+\right)}^{*}$ of a dual model without constraints; furthermore by Lemma $2 \omega_{\left(\beta, \mathbf{K}^{*} ;+\right)}^{*}=\lim _{\mathbf{K}^{* * \rightarrow K^{*}}} \omega_{\left(\beta, \mathbf{K}^{*} ;+\right)}^{*}$ which concludes the proof since $\omega_{\left(\beta, \boldsymbol{K}^{\prime *} ;+\right)}^{*}$ is related to $\omega_{\left(\beta, \mathbf{K}^{\prime} ; 0\right)}$ and $K_{\boldsymbol{B}_{\infty}}^{*}>0 \mathrm{implies}$ $K_{B_{\infty}}^{\prime} \rightarrow+\infty$.

\section{Example}

In the following examples, we shall use the above results to discuss the number of phase transitions associated with breakdown of symmetry; we could obtain the same informations using the symmetry properties of the systems [3].

1) Consider the triangular lattice with 3-body forces between nearestneighbours

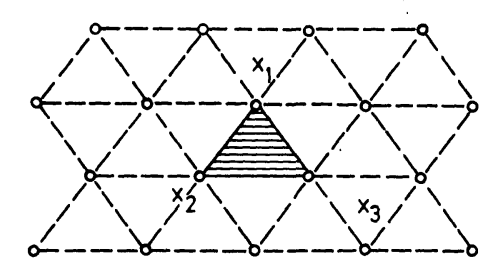

$$
\begin{aligned}
& \{\circ\}=\mathscr{L} \\
& \left\{\begin{array}{l}
\text { 只 } \\
0 .-\circ
\end{array}\right\}=\mathscr{B}
\end{aligned}
$$

$$
\mathscr{P}_{f}(\mathscr{L})=\overline{\mathscr{B}} \cup x_{1} \overline{\mathscr{B}} \cup x_{2} \overline{\mathscr{B}} \cup x_{3} \overline{\mathscr{B}}
$$

$\overline{\mathscr{B}}$ is the subgroup of $\mathscr{P}_{f}(\mathscr{L})$ generated by finite products of the elementary "triangles".

It follows from Theorem 4 above that there exists $\beta_{i}, i=1,2,3$ such that:

$$
\omega_{+}\left[\sigma_{x_{i} \bar{B}}\right]=\left\{\begin{array}{rr}
0 & \beta<\beta_{i} \\
>0 & \beta>\beta_{i}
\end{array}\right.
$$

and

$$
C_{i, 1} \omega_{+}\left[\sigma_{x_{i}}\right] \leqq \omega_{+}\left[\sigma_{x_{i} \bar{B}}\right] \leqq C_{i, 2} \omega_{+}\left[\sigma_{x_{i}}\right]
$$


However, since $\omega_{+}$is $\mathbb{Z}^{2}$-invariant it follows that $\beta_{1}=\beta_{2}=\beta_{3}$ and therefore there exists a unique temperature $\beta_{c}$ where the symmetry is completely broken, and only one order parameter associated with the correlation function: i.e. all equilibrium states are symmetric for $\beta<\beta_{c}$ and $\mathscr{S}_{+}=\{\phi\}$ for $\beta>\beta_{c}$.

It thus follows that the critical indices of the different order parameters $\omega_{+}\left[\sigma_{x_{i} \bar{B}}\right]$ are the same; in particular $\omega_{+}\left[\sigma_{x_{1}}\right]$ and $\omega_{+}\left[\sigma_{x_{1} x_{2}}\right]$ have the same critical indices, i.e. the spontaneous magnetization and the spontaneous polarization (nearest neighbour pair correlation function) have the same critical index ( $b=1 / 12$ [7]).

2) Let us now consider the Ashkin-Teller model defined by means of two square Ising models $\mathscr{L}_{1}, \mathscr{L}_{2}$ coupled by 4-body forces
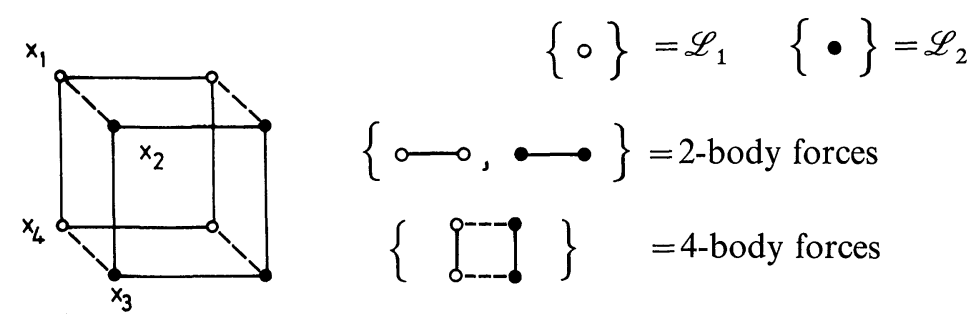

$\mathscr{P}_{f}(\mathscr{L})=\overline{\mathscr{B}} \cup x_{1} \overline{\mathscr{B}} \cup x_{2} \overline{\mathscr{B}} \cup x_{1} x_{2} \overline{\mathscr{B}}$

where

$$
\begin{aligned}
\overline{\mathscr{B}} & =\left\{\bar{B} \in \mathscr{P}_{f}(\mathscr{L}) ;\left|\bar{B} \cap \mathscr{L}_{1}\right|=\text { even }\left|\bar{B} \cap \mathscr{L}_{2}\right|=\text { even }\right\} \\
x_{1} \overline{\mathscr{B}} & =\left\{X \in \mathscr{P}_{f}(\mathscr{L}) ;\left|X \cap \mathscr{L}_{1}\right|=\text { odd }\left|X \cap \mathscr{L}_{2}\right|=\text { even }\right\} \\
x_{2} \overline{\mathscr{B}} & =\left\{X \in \mathscr{P}_{f}(\mathscr{L}) ;\left|X \cap \mathscr{L}_{1}\right|=\text { even }\left|X \cap \mathscr{L}_{2}\right|=\text { odd }\right\} \\
x_{1} x_{2} \overline{\mathscr{B}} & =\left\{X \in \mathscr{P}_{f}(\mathscr{L}) ;\left|X \cap \mathscr{L}_{1}\right|=\text { odd }\left|X \cap \mathscr{L}_{2}\right|=\text { odd }\right\} .
\end{aligned}
$$

It then follows from Theorem 4 that there exist, $\beta_{1}, \beta_{2}, \beta_{12}$ such that

$$
\begin{gathered}
\omega_{+}\left[\sigma_{x_{1} \bar{B}}\right]=\left\{\begin{aligned}
0 & \beta<\beta_{1} \\
>0 & \beta>\beta_{2}
\end{aligned}\right. \\
\omega_{+}\left[\sigma_{x_{1} \bar{B}}\right]=\left\{\begin{aligned}
0 & \beta<\beta_{2} \\
>0 & \beta>\beta_{2}
\end{aligned}\right. \\
\omega_{+}\left[\sigma_{x_{1} x_{2} \bar{B}}\right]=\left\{\begin{array}{rl}
0 & \beta<\beta_{12} \\
>0 & \beta>\beta_{12}
\end{array} .\right.
\end{gathered}
$$

Let us assume that $\beta_{1}=\min \left\{\beta_{1}, \beta_{2}, \beta_{12}\right\}=\beta_{c}$ then it follows from

$$
\begin{aligned}
& \omega_{+}\left[\sigma_{x_{1} x_{2}}\right] \geqq \omega_{+}\left[\sigma_{x_{1}}\right] \omega_{+}\left[\sigma_{x_{2}}\right] \\
& \omega_{+}\left[\sigma_{x_{2}}\right] \geqq \omega_{+}\left[\sigma_{x_{1}}\right] \omega_{+}\left[\sigma_{x_{1} x_{2}}\right]
\end{aligned}
$$

that $\beta_{2}=\beta_{12}$. 
Therefore, there can be at most two critical temperatures where the symmetry can be broken (in fact renormalization group arguments [9] indicate that for certain values of the parameters $\beta_{1} \neq \beta_{2}$ ), and at most three distinct critical indices associated with correlation functions which are given by

$$
p_{1}(\beta)=\omega_{+}\left[\sigma_{x_{1}}\right] \quad p_{2}(\beta)=\omega_{+}\left[\sigma_{x_{2}}\right] \quad p_{3}(\beta)=\omega_{+}\left[\sigma_{x_{1} x_{2}}\right]
$$

In particular $\omega_{+}\left[\sigma_{x_{1}}\right], \omega_{+}\left[\sigma_{x_{1} x_{2} x_{3}}\right]$ and $\omega_{+}\left[\sigma_{x_{2} x_{3} x_{4}}\right]$ have the same critical indices.

To conclude the discussion of these examples, we remark that the reduction in the number of critical temperatures do not have the same origin in both examples; in the first example it follows from the fact that there exists $X_{i}$ and $X_{j}$ such that $X_{j}=X_{i}+a$, in the second example from the fact that there exists $X_{i}, X_{j}, X_{k}$ which are related by $X_{k}=X_{i} X_{j}$.

\section{Coexistence of Phases in Ising Ferromagnet}

Let $\{\mathscr{L}, K=\beta J, \mathscr{B}\}$ be a $\mathbb{Z}^{v}$-invariant ferromagnetic system with finite range interactions as defined in Section 2. The purpose of this section is to show that there exists a unique $\mathbb{Z}^{v}$-invariant, symmetric, equilibrium state whenever the energy per site is a continuous function of the temperature. The proof of this results follows exactly the proof given in [2] and we shall only indicate the necessary modifications.

We know that for the systems we are considering the infinite volume Gibbs states $\omega_{(\beta, J ;+)}$ is well defined, $\mathbb{Z}^{v}$-invariant. Furthermore using the fundamental inequality of [2], it is sufficient to show that

$$
\omega_{(\beta, J ;+)}\left[\sigma_{B}\right]=\bar{\omega}_{(\beta, J)}\left[\sigma_{B}\right] \quad \forall B \in \mathscr{B}
$$

for all $\mathbb{Z}^{v}$-invariant equilibrium states to conclude at the unicity of the $\mathbb{Z}^{v}$ invariant, symmetric, equilibrium state [2].

Theorem 7. Let $\{\mathscr{L}, K=\beta J, \mathscr{B}\}$ be a $\mathbb{Z}^{v}$-invariant, ferromagnetic system with finite range interactions. If $\frac{\partial \psi(\beta, J)}{\partial \beta}$ exists, i.e. the energy per site is continuous, then there exists a unique $\mathbb{Z}^{v}$-invariant symmetric equilibrium state.

Proof. With $\mathscr{B}_{0}$ a fundamental set of bonds $B \ni\{0\}$, i.e. $\forall B \in \mathscr{B}, \exists a \in \mathbb{Z}^{v}$ and $B_{0} \in \mathscr{B}_{0}$ such that $B=B_{0}+a$, then by the general arguments of [2] $\frac{\partial \psi(\beta, J)}{\partial \beta}$ continuous implies that for any $\mathbb{Z}^{v}$-invariant equilibrium state $\bar{\omega}$

$$
\frac{\partial \psi}{\partial \beta}=\sum_{B \in \mathscr{B}_{0}} J(B) \bar{\omega}_{(\beta, J)}\left[\sigma_{B}\right]=\sum_{B \in \mathscr{B}_{0}} J(B) \omega_{(\beta, J ;+)}\left[\sigma_{B}\right] .
$$

But

$$
\omega_{(\beta, J ;+)}\left[\sigma_{B}\right] \geqq \bar{\omega}_{(\beta, J)}\left[\sigma_{B}\right]
$$

implies

$$
\omega_{(\beta, J ;+)}\left[\sigma_{B}\right]=\bar{\omega}_{(\beta, J)}\left[\sigma_{B}\right] \quad \forall B \in \mathscr{B}_{0} .
$$


Since $\omega_{(\beta, J ;+)}$ is $\mathbb{Z}^{v}$-invariant and since $\mathscr{B}_{0}$ is generating for $\mathscr{B}$ it follows that Equation (8) holds for all $B$ in $\mathscr{B}$ which concludes the proof because of the above remark.

Using the results mentioned in Section 2, we have immediately the following property:

Corollary 8. Let the conditions of Theorem 7 hold. If $\frac{\partial \psi}{\partial \beta}$ exists, then there exists exactly $|\mathscr{S}| \mathscr{S}_{+} \mid$extremal equilibrium states which are invariant under some translation subgroup $\mathscr{T}$ with $\left|\mathbb{Z}^{v} / \mathscr{T}\right|<\infty$ where $\mathscr{S}$ and $\mathscr{S}_{+}$are respectively defined by Equations (1) and (2).

Remark. Using the convexity property of $\psi(\beta)$ it follows that for all but a countable values of temperatures there exists a unique equilibrium state with the same symmetry group as the hamiltonian.

\section{Example: Fluid-Crystal Transition}

Let us consider the ferromagnetic system of Example 1, Section 3 together with an external field (1-body interaction) $h \gtrless 0$. It has been shown in [3, p.92] that there coexists at $h=0$, and low temperatures, at least two phases $\omega_{+}, \omega_{s}$ which decompose into $1+3$ extremal equilibrium states, namely $\omega_{+}$which is translation invariant and three other states which are not translation invariant. They correspond to a "fluid" phase and a "solid" phase. We also know [3] that for $0>h>-J$ there exists a phase $\omega_{s}$ which decomposes into 3 extremal states and is thus interpreted as the "solid" phase, while for $h>0$ we know that there exists the state $\omega_{+}$which is translation invariant and thus corresponds to the "fluid" phase.

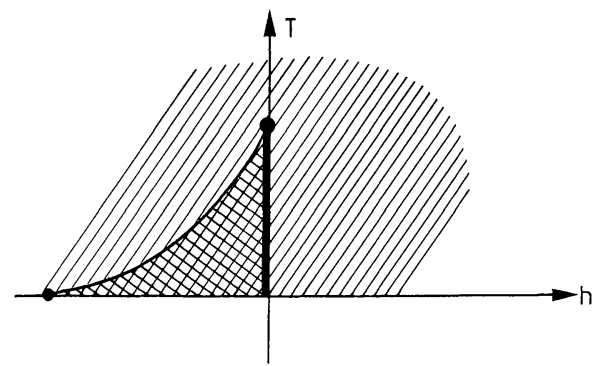

a)

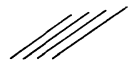

b)

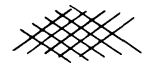

c)

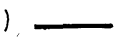

$: 1$ pure phase which is an extremal equilibrium state thus corresponds to the "fluid phase".

:1 pure phase which decomposes into 3 extremal equilibrium states which are not translationally invariant and thus correspond to "solid phase".

: coexistence between solid phase and fluid phase, there exists two pure phases which decompose into 4 extremal equilibrium state, 1 invariant under translation and 3 which are not. 
To conclude at the existence of a phase transition of the type "fluid-solid" we would like to know that there exists for all $(h, T)$ a unique $\mathbb{Z}^{2}$-invariant, symmetric, equilibrium state; indeed from such a result would follow that

the "fluid" phase is the only phase at $h>0$

the "solid" phase is the only phase at $h<0$

there coexists exactly two phases namely the "fluid" and the "solid" at $h=0$.

Using the above Theorem 7, we can conclude that if the energy per site is continuous in $T$ for any $h$ around $h=0$ then the phase transition which occurs around $h=0$ is of the "fluid-solid" type, and the phase diagram is as expected [3]:

Acknowledgements. We thank J. Slawny for many useful comments and for calling to our attention that the equivalent of Theorem 7 and Corollary 8 were noted independently by J. Bricmont in his thesis (Louvain, 1977).

\section{References}

1. Lebowitz,J.L.: On the equivalence of different order parameters for ising ferromagnet. J. Stat. Phys. 16, 3 (1977)

2. Lebowitz,J.L.: Coexistence of phase in ising ferromagnet. J. Stat. Phys. 16, 463 (1977)

3. Gruber,C., Hintermann, A., Merlini,D.: Group analysis of classical lattice systems. Lecture notes in physics, Vol. 60. Berlin-Heidelberg-New York: Springer 1977

4. Lebowitz,J.L.: Number of phases in one component ferromagnet. Preprint (1977)

5. Griffiths, R. B.: J. Math. Phys. 8, 478 (1967)

Kelley,D.G., Sherman,S.: J. Math. Phys. 9, 466 (1969)

6. Ruelle,D.: Statistical mechanics. New York: Benjamin 1965

7. Baxter, R.J., Sykes, M.F., Watts,M.G.: J. Phys. A 8, 245 (1975)

8. Slawny,J.: Commun. math. Phys. 35, 2971 (1974)

9. Knops,H.J.F.: Branch point in the critical surface of the Ashkin-Teller model in the renormalization group theory. Preprint (1976)

Communicated by E. Lieb

Received October 15, 1977 